\title{
Reflexiones en torno a la didáctica de la teoría literaria
}

\author{
Ladys Jiménez \\ Profesora de Literatura \\ Programa de Lengua Castellana \\ ladys.jimenez@usco.edu.co
}

Resumen: la temática se desarrolla en torno a la didáctica de la Teoría Literaria, intenta proponer una reflexión de la enseñabilidad de ésta en el ámbito universitario. Seguidamente hay una aproximación a la teoría literaria, la pedagogía y la didáctica, se plantean algunas dificultades metodológicas. Por último se esboza una propuesta acerca de su didactización.

Palabras claves: Teoría literaria, didáctica, literatura, saber pedagógico, saber disciplinar, teoría, práctica, enseñabilidad.

En los últimos años la reflexión entre el saber pedagógico y el saber disciplinar se debate en el contexto universitario especialmente en las Facultades de Educación. Por un lado están los que consideran que "la formación de maestros debe hacerse fundamentada en un alto dominio de las ciencias, y por consiguiente, consideran que lo secundario en la formación de maestro es el saber de la pedagogía y la didáctica (...) sólo el dominio del saber disciplinar forma el criterio de los enseñantes" (Ibarra, 2010). Por otra parte están los que piensan que "la base fundamental de la enseñanza es la pedagogía y didáctica como su discurso práctico, y que por lo tanto, sólo el dominio de un conocimiento científico o técnico no determina la criteriología, ni las habilidades ni destrezas que provee la inteligencia pedagógica a la práctica educativa" (Ibarra, 2010) Alrededor de esta dos posturas se debate el papel del docente como intermediario entre la teoria y la práctica.

Estas dos posiciones opuestas y hasta contradictorias frente a la práctica educativa se resuelven en la medida que los conocimientos determinan y articulan la práctica y viceversa. Desde ese marco de referencia parte esta reflexión, que integra el conocimiento profundo de la disciplina con un dominio sobre cómo se enseña, además se intenta dilucidar el papel del docente como intermediario entre las ideas abstractas y su aplicación concreta, que permite fomentar una interacción apropiada de los contenidos teóricos y facilitar el aprendizaje.

En ese sentido el propósito de este texto es contribuir a la reflexión que integra el saber disciplinario y el saber pedagógico ${ }^{124}$,

${ }^{124}$ El saber pedagógico no se dirige a determinar el valor cientifico de la pedagogia y su fundamento lógico, sino que permite establecer puentes caminos y encuentros con otros saberes y aún con disciplinas formalmente constituidas. Ibarra, Saber disciplinar y saber pedagógico ¿convergencia o divergencia?, publicado en revista Paideia, No 15, Facultad de Educación, universidad Surcolombiana, Neiva 2010. 
específicamente a la didactización de la teoría literaria en el contexto universitario, a establecer su relevancia en el ámbito de la práctica docente. Para esto se realizará una breve presentación sobre la didáctica y la teoría literaria, seguidamente se abordarán algunas dificultades metodológicas en para abordar su estudio y por último se esboza una propuesta acerca de la didáctica de la de la teoría literaria.

\section{Teoría Literaria y su relación con la Pedagogía y la Didáctica}

Se conoce como Teoría Literaria ${ }^{125}$ a "la reflexión teórica sobre el sistema de aspectos constantes y específicos en los textos. Su cometido es esclarecer los fundamentos teóricos de la Ciencia Literaria y en concreto, construir un metalenguaje a partir del cual sea posible estudiar con rigor cualquiera de las cuestiones que se formulen alrededor del hecho literario" (Josep Ballester: 2005) Su objetivo principal sería el de desentrañar y evidenciar las leyes generales que subyacen en el texto literario. La Teoria Literaria nace con el Formalismo ruso "en el momento en que hemos sido capaces de especificar un objeto propio: el lenguaje literario y la ambición de construir una ciencia que explicara el modo de ser y de comportarse de la Literatura en tanto lenguaje" (Pozuelo Yvancos, 1988)

En el desarrollo de esta disciplina han intervenido una serie de escuelas o tendencias literarias como: El formalismo ruso ${ }^{126}$ considera que la literatura constituye el objeto de estudio de la ciencia literaria y analiza cómo funcionan en el texto los principios y mecanismos de construcción.

El New Criticism ${ }^{127}$ propone el desarrollo de una crítica inmanente centrada en el análisis de las formas literarias, "la densidad de la semántica del lenguaje literario frente a otras formas de discurso lingüistico; se introduce de este modo la noción de polivalencia semántica, que, afín a la de ambigüedad", (Maestro, 1985) estudiosos de la estética y la semántica literaria conciben al texto literario "como un todo orgánico, autónomo y autosuficiente, en el que los diferentes elementos se disponen de modo estructural, formando un sistema de relaciones y unidades formales solidarias entre sí." (Maestro, 1985).

El estructuralismo una de las corrientes criticas y teóricas más relevantes del siglo XX, considera el texto literario como una estructura que se

\footnotetext{
${ }^{125}$ A lo largo de la historia la Teoria Literaria se conoce con diversos nombres: Arte Poética (Aristóteles y Horacio), Teoria general de las formas literarias (Genete) pero en la actualidad se conoce como Teoria Literaria (Tomacheski, R Wellek y A. Warren, T. Todorov, entre otros)

${ }^{126}$ Del formalismo los estudios reconocen dos etapas representativas, la primera inicia(1914-1920) con la publicación del ensayo "el arte como recurso" de Viktor Shklovi en esa etapa los formalistas desarrollan un intenso trabajo sobre la definición de lo literario, el estudio de la métrica y el lenguaje literario. La segunda etapa (1921 - 1930) Vladimir Propp, Juri Tinianov, y Roman Jakobson con Problemas del estudio de la literatura y la lengua "resumen las posiciones fundamentales de la última fase del formalismo y contienen al mismo tiempo los gérmenes de las teorias del estructuralismo checo" (Fokeman, 1984, 27) y desarrollan investigaciones sobre propiedades estilisticas y estructuras formales sobre la novela, el cuento y los motivos literarios.

127 "New Criticism es la denominación que recibe un grupo de estudiosos norteamericano que, durante el segundo cuarto del presente siglo, desarrollaron, en las universidades de los Estados Unidos, un movimiento de investigación literatia de gran importancia en la configuración actual de la modernd teoria de la literatura. Esta denominación procede del titulo de una obra del poeta y crítico norteamericano ]. Crowe -The New Criticism (1941), que constituye un estudio critico sobre la obra de T.S. Eliot, I.A. Richards e Y. Winters. Jesús G. Maestro - Introducción a la teoria de la literatura en:

ht t p://academiaeditorial. com/cms/uploa/pdf/Critica\%20heterodoxa/ 053\%20\%20Introduccion\%20a\%20la\%20teoria\%20de\%20la\%20literatura\%20-\%20Siglo\%20XX.pdf [consulta enero 26 de 2010]
} 
puede descomponer en varios niveles de análisis, Según Bernstein la obra de arte sólo puede funcionar signo a causa de su estructura, la cual se puede analizar en factores reconocibles.(Fokeman, 1991). El estructuralismo aplica la teoría lingüística al análisis del texto literario, se interesa en el análisis de las estructuras de la obra literaria.

Teorias sociológicas de la Literatura: concibe la obra literaria como textos basados en "estructuras mentales transindividuales", pertenecientes a grupos o clases sociales. Lucien Goldman uno de los principales exponentes de la sociología clásica de la literatura, planteó el concepto de visión de mundo o cosmovisión como instrumento primordial e indispensable para entender las expresiones artísticas de los individuos; más exactamente, la definió como un conjunto de ideas y sentimientos propios de un grupo que queda así determinado en un momento especifico de la historia y del desarrollo social. Posteriormente Mijail Bajtín da inicio a lo que conocemos como la tradición sociocrítica, que considera toda expresión de arte como evaluación del mundo y plantea el concepto de forma arquitectónica ${ }^{128}$. La sociocrítica, analiza los procesos críticos que se generan a partir de una obra artística, por lo cual prefiere textos críticos y dialógicos. El texto literario no es la afirmación de una ideología ya existente, sino la deconstrucción y desestabilización de la misma (Pouliquen, 1995).
La teoría de la recepción: considera al lector como centro de las principales reflexiones sobre el texto, la interpretación y lectura se configuran como procesos de cooperación de sentido de la obra literaria. Estas y otras teorías ${ }^{129}$ plantean pluralidad de lecturas, enfoques y métodos de análisis de las que puede ser objeto el discurso literario.

Pedagogía y Didáctica: la didáctica como campo de saber conoce un lugar histórico ligado a la historia de la pedagogía, la cual:

constituye el componente conceptual desde el cual, y con la finalidad de formar a los sujetos, pueden los maestros articular de manera creativa los discursos específicos de las ciencias, de la ética y de la estética con los valores propios de la cultura para hacer posible que autónomamente el sujeto se apropie del conocimiento, en un proceso de desarrollo humano que implica su inteligencia, sus sentimientos y sus prácticas, jalonadas por una tensión de perfeccionamiento continuo, autónomo e integral (Ibarra, 2010)

Como campo de saber humanizante, la pedagogía ha aportado a la interpretación y comprensión del hecho educativo y por ende a la comprensión de su modo práctico: la didáctica ${ }^{130}$, la cual se define como una "ciencia que tiene por objeto la organización y orientación de situaciones de enseñanza-aprendizaje de

\footnotetext{
${ }^{128}$ La forma arquitectónica definida por Bajtín como el material lingüistico en la obra de arte con determinado contenido axiológico o conocimiento del mundo-, y ésta a su vez está conformada por la forma composicional. Para Bajtín, el contenido de la obra de arte siempre hace referencia al conocimiento del mundo. Con la forma, lo que se hace es dar una valoración del mundo en la unificación y organización de los valores cognoscitivos que se llevan a cabo mediante la forma composicional a través del material verbal.

${ }^{129}$ Otras teorias como la semiótica, la obra como signo y la psicocritica y la mitocrítica son abordadas por teóricos como Fokkeman, Eagleton y Pozuelo Yvancos René Welker entre otros.

${ }^{130}$ La didáctica toma la enseñanza como objeto central de sus elaboraciones en tanto pretende describir y explicar las prácticas o situaciones de enseñanza, Edith Litwin Las Configuraciones Didácticas: una Nueva Agenda para la Enseñanza Superior, Buenos Aires, Paidós.
} 
carácter instructivo, tendentes a la formación del individuo en estrecha dependencia de su educación integral". (Escudero 1980).

Para Jaime Jaramillo Uribe, la didáctica "se ocupa de los métodos más adecuados para transmitir un acervo cultural o cientifico. Es la parte metodológica de la pedagogia, la más estrictamente científica y la que se apoya en la lógica y la teoria de las ciencias (Uribe,1990). Por lo anterior se puede afirmar que la didáctica es el discurso a través del cual el saber pedagógico ha pensado la enseñanza hasta hacerla el objeto central de sus elaboraciones. Se ubica en un contexto teórico transdisciplinario de las disciplinas que han ofrecido diversos enfoques de acercamiento y análisis.

En la actualidad existen tres posturas sobre la naturaleza del conocimiento didáctico. La primera alude a la didáctica como parte de una teoría de la educación ${ }^{131}$. La segunda plantea la constitución de didácticas específicas ${ }^{132}$ con investigación sobre su objeto- la enseñanza. Desde esta línea, se toman aportes de disciplinas relacionadas con la didáctica (la psicología, ciencia cognitiva y el saber disciplinar). Por último, una tercera postura niega la existencia de la didáctica como disciplina, pues no cuenta con conceptos propios y más bien se define como un conjunto de reflexiones sustentadas en diferentes ciencias como la psicologia del aprendizaje. Con base en lo anterior esta reflexión se sustenta en los supuestos de la segunda postura: la constitución de las didácticas especificas, que analiza la relación entre el saber especifico y el saber disciplinar y su articulación entre estudiantes, docentes y los contenidos de la disciplina. A partir de esos presupuestos se observa la enseñanza de la Teoria y Literaria y se plantean algunas propuestas.

La enseñanza de la Teoria Literaria como estudio científico plantea los recodos de la estructura y funcionamiento del texto literario, además expone concepciones de interpretación, "sentido" y recepción de la obra literaria. Su relación con el saber pedagógico permite la lectura humanizada del discurso teórico, en ese sentido la didáctica se constituye en medio de apropiación reflexión y evaluación del saber disciplinar "implica la formación del sujeto racional que reflexiona y crea la posibilidad de continuidad de un saber cientifico indispensable para la vida social, y de responsabilidad ética y política en el uso social del conocimiento" (Ibarra, 2010).

\section{Algunas dificultades metodológicas en la enseñanza de la Teoría Literaria:}

Desde la experiencia de docente universitaria la enseñanza de la Teoría Literaria plantea algunos problemas metodológicos que van desde el dominio de la disciplina hasta la didactización de esta. El primero de ellos se relaciona con la multiplicidad de teorias que se han generado para abordar el análisis del texto literario, esto delimita el estudio profundo de cada una de las teorias. Sumado a esto cada una de las teorías ofrece distintos métodos de acercamientos teóricos críticos.

Un problema metodológico se relaciona con la organización de los contenidos de la disciplina. Pues el estudio de la Teoría Literaria en el

\footnotetext{
${ }^{131}$ Camilloni, A. (1994). "Epistemología de la didáctica de las ciencias sociales". En Aisenberg, B. y Alderoqui S. Didáctica de las Ciencias Sociales. Aportes y Reflexiones. Buenos Aires. Paidós. Pág. 27

${ }^{132}$ En la actualidad algunas didáctica ya se encuentran constituidas (Didácticas de enseñanza de las lenguas, didáctica de la Lingüística, Didáctica de la Literatura) o en proceso de constitución.
} 
contexto universitario alude por lo general a dos cursos introductorios, en el cual la aproximación a los contenidos se realiza desde una visión panorámica de las principales teorías.

Otro problema en la enseñanza de la Teoria Literaria en su aplicación práctica alude al encuentro con otra disciplina la Crítica Literaria $^{133}$. Además de lo anterior surgen los siguientes interrogante ¿cómo se abordan el conjunto de elementos culturales y sociales que tienen alguna relación con la génesis de Teorías Literarias?, ¿cuáles serian las tareas concretas de la teoría literaria frente a la didáctica de la literatura?

Con relación a la didáctica uno de los problemas metodológicos de la enseñanza de la Teoría Literaria y de otras disciplinas es la creencia común de que basta con saber la disciplina para poder enseñarla. Esta discusión ha sido un punto álgido en el ámbito pedagógico en la medida que la enseñabilidad de la misma no se desprende del saber teórico, sino más bien un conjunto de elementos que integran las intencionalidades curriculares, docentes, estudiantes y contenidos.

Otro problema se encuentra asociado con la errada idea de la didáctica como reductora de contenidos en el que "las ideas han sido objeto de simplificaciones que las han reducido a meras versiones desvirtuadas de las ideas originales aparecida en los contextos de investigación de donde han sido adoptadas, y a menudo se asemejan muy poco" (Widdowson, 1998) La didáctica implica las transformaciones adaptativas de los contenidos para su enseñanza pero estos cambios no deben implicar pérdida o disminución del saber disciplinar.

\section{Propuesta metodológica didáctica de la Teoría Literaria}

Como propuesta metodológica retomamos los planteamientos propuestos Widdowson sobre la pragmática de la pedagogía de lenguas. Este autor propone un modelo de mediación en el que distingue dos fases: a la primera llama apreciación: se centra en la teoría y consiste en la interpretación de las ideas haciendo uso de sus propios términos de referencia, en el contexto de la propia génesis teórica, y en la evaluación y pertenecía o validez teórica de estas ideas en relación con el campo de investigación del contexto que es su aplicación, por lo tanto la evaluación consiste en especificar lo que podríamos denominar el valor de la transferencia de ideas. (Widdowson, 1998)

De esta propuesta se retoman algunos aspectos importantes válidos en la reflexión sobre la didáctica de la Teoría Literaria. Según este autor la didáctica retoma actores inmersos en el proceso de enseñanza: el docente como mediador de contenidos interpreta ideas y abstracciones del constructo teórico pero en sus propios términos de referencia, por ejemplo al trabajar con el concepto de visión de mundo que propone Lucien Goldmann, el docente como mediador además de los planteamientos teóricos de la génesis del concepto, debe considerar los procesos subjetivos y sociales implícitos, ya que estos se convierten en aspectos relevantes para la evaluación y la validez teórica de las ideas en su aplicación.

\footnotetext{
${ }^{133}$ Según Bobes Naves la Critica literaria se ocuparia del análisis de autores y obras concretas, insistiendo más en una valoración, de orden literario, lingüistico, filosófico, histórico, sociológico, etc. Mientras que la Teoría de la Literatura se ocupa del conocimiento cientifico de las obras literarias, conocimiento que pretende en su aplicación una validez general, mediante procedimientos inductivos. MAESTRO, Jesús $\mathrm{G}$ (1984). Introducción a la Teoría de la literatura. Internet: http://mirabeleditorial.com/pdf/055\%20-\%20ITL6\%20-\%20Novela.pdf (consulta: 27 de enero de 2011) pág. 11
} 
El segundo proceso pragmático el autor lo llama aplicación: un proceso cercano a la práctica y es concebida en dos fases: en la primera las ideas son sometidas a la operación en el terreno práctico, por ejemplo una actividad que podría proponerse después de conocer los tipos de narradores que plantea Todorov, es el análisis del discurso literario y reconocer el tipo de narrador y su sentido en el texto. Lo que se pretende es aplicar las teorías del narrador y llevarlas al terreno práctico. En la segunda la evaluación valora las consecuencias de las mismas: comprensión de las abstracciones y conceptos trabajados en clase, y luego la aplicación de estos ya sea en la construcción de hipótesis hipotéticas deductivas en el que el estudiante llevará las teorias al terreno de la aplicación. En este caso la evaluación sirve para establecer el efecto práctico de las ideas sometidas a la operación (Widdowson, 1998)

Apreciación es por tanto una evaluación conceptual basada en la adecuada comprensión y sirve para establecer un conjunto de principios válido de pertenencia general (Widdowson, 1998). La apreciación consiste en evaluación de los contenidos a partir de diferentes tipos de actividades que den cuenta de comprensión de categorias de análisis como narrador, focalización, lector modelo, entre otros. La aplicación es evaluación empírica basada en la experiencia del docente e interviene en el diseño de técnicas eficaces, especificas de unas circunstancias concretas. (Widdowson, 1998) Actividades de comprensión lectora, análisis de casos a partir del discurso literario, construcción de hipótesis entre otras.

La preparación metodológica parte de la estabilidad del ámbito teórico y práctico en el que se enmarca. Sólo la comprensión de los conceptos hace posible una aplicación versátil. Por tanto la enseñabilidad de la Teoría Literaria apuntaría especialmente al desarrollo de actividades que propicien la comprensión: proponer estrategias de aprendizajes encaminadas a la lectura, análisis y entendimiento de los contenidos, propiciar el desarrollo del intelecto a través de ejercicios de razonamiento e interpretación que vayan encaminadas al conocimiento, pues sólo la comprensión de los contenidos posibilitaría la buena aplicación de estos.

Sin embargo la mediación puede fracasar, frente lo anterior el autor afirma que: "es plausible una evaluación de la validez, positiva o negativa, que no haya partido de una interpretación correcta de las ideas. Esto puede llevar a una aceptación apasionada o a un rechazo no avalado por una comprensión acertada de las ideas, a la imposición de una manera de pensar sobre la otra" aunque cabe anotar que una aceptación o imposición no escapa a "las hondas estructuras de persuasión ${ }^{134}$, la imposición de una teoría sobre la otra, una aceptación apasionada o un rechazo además de estar vinculadas a una interpretación errónea de las ideas, también puede estar relacionada con "juicios de valor que se relacionan estrechamente con las ideologias sociales." (Eagletón pág.10) Un ejemplo desde la experiencia docente es ver como algunos estudiantes se sienten más cómodos con unas teorias que con otras. La razón de esto puede deberse: por un lado a la errónea comprensión e interpretación de los contenidos. Por otro lado puede estar relacionado con lo persuasivo y los juicios de valor en relación con las ideologias sociales desde las cuales se hace la lectura de las teorías.

\footnotetext{
${ }^{134}$ Eagletón afirma que lo que conocemos literatura dichos juicios de valor no tienen nada de caprichosos. Tienen raices en hondas estructuras de persuasión, Una introducción a la teoria literaria,
} 
Una didáctica de la Teoría Literaria además de la comprensión de los contenidos de la disciplina, implicaría el aprovechamiento de otros insumos. Un ejemplo son las reflexiones que realizan escritores acerca de su ejercicio literario. Julio Cortázar, Mario Vargas Llosa, Gabriel Garcia Márquez producen Teoría Literaria, a partir de la reflexión del proceso de creación hacen teorias del discurso literario. Cartas a un joven novelista sería una muestra de ejercicio didáctico.

En relación con el estudiante se propone incentivar la competencia literaria, concebida como una competencia lectora, con estrategias que permitan al lector la construcción del sentido y la comprensión e interpretación de textos, así como la expresión creativa mediante la manipulación y producción de textos (Prado, 2004: 333). A partir de la lectura y comprensión de los contenidos se busca desarrollar competencias literarias a partir de la comprensión lectora, la adquisición y aplicación del léxico especializado, construcción de hipótesis, ensayos que den cuenta de los procesos de comprensión y aplicación.

Para Widdowson los maestros como intermediarios y agentes de renovación tendíamos la tarea de enseñar la teoría en relación con las conexiones de la realidad y con contextos concretos de clase. Se parte de conocimientos previos de los estudiantes y se articulan con los nuevos contenidos por enseñar. Por tanto se parte de una concepción activa del aprendizaje, en la reconstrucción y contextualización del conocimiento.

Una metodología que aportar las condiciones para que esta adquisición sea posible que se propone desde la pedagogía critica a través de procesos de reflexión y análisis de la teoría y la práctica por parte de los actores implicados en el proceso pedagógico tal como lo afirma Aranguren: "el nexo de la teoría y la práctica en lo social-educativo ha de entenderse conceptualmente como actividad práctica objetiva y transformadora en respuesta a necesidades sociales y no meramente científico pedagógicas, pues entre la teoria y la práctica existe un continuo transitar mediado por la conciencia social".

Finalmente esta reflexión apunta a la necesidad de revisar los procesos de enseñabilidad de la Teoría Literaria y proponer a partir de la experiencia docente una didáctica que permita mejorar los procesos de enseñanza y aprendizaje. Asimismo examinar las metodologías y maneras de cómo relacionamos conocimientos previos con los nuevos por enseñar.

No cabe duda que en la enseñabilidad de la Teoría Literaria intervienen múltiples factores, en ocasiones inapreciables. El reconocimiento de otras disciplinas como la sociología, la lingüistica y psicología y la relación de interdisciplinariedad de la Teoría Literaria con estas disciplinas. Además es importante rescatar las aportaciones de la Teoría Literaria en el desarrollo de la Competencia Literaria.

De este modo no olvidamos que ésta es solo una reflexión que apunta al ejercicio docentes de proponer actividades encaminadas a desarrollar en el estudiante conocimientos, habilidades, destrezas y actitudes en miras de lograr la comprensión, la aplicación y finalmente evaluación de los contenidos propuestos en una formación que vincule la teoría con la práctica.

\section{Bibliografía}

ARANGURREN Carmen R. "Teoria y praxis en la enseñanza de la historia: una relación 
epistemológica", Educere, enero marzo año, vol. 9 número 028 Universidad de los Andes Mérida, Venezuela pág 61-65

CAMILLONI, A. "Reflexiones para la construcción de una Didáctica para la Educación Superior". Ponencia presentada en las Primeras Jornadas trasandinas sobre planeamiento, gestión y evaluación "Didáctica de Nivel Superior" Universitaria. Chile. 19 y 20 de enero 1995.

MENDOZA, Fillola, A. (coord.): Conceptos clave en Didáctica de la Lengua y la Literatura, Sociedad Española de Didáctica de la Lengua y la Literatura-ICE de la Universidad de BarcelonaHorsori, Barcelona, 1998.
PRADO Aragonés, Josefina. Didáctica de la lengua y la literatura para educar en el siglo XXI. Madrid, Editorial La Muralla, 2000.

POULIQUEN, Helena. Para una poética sociológica, Serie Cuadernos de Trabajo, No 12 , Bogotá, Facultad de Ciencias Humanas, Universidad Nacional de Colombia, 1995.

FOKKEMAN, D. W., IBSCH, E. Teorias de la literatura en el siglo XX, Madrid, Cátedra, 1981. POZUELO YVANCOS, J. M". Teoría del Lenguaje Literario, Madrid, Cátedra, 1988.

CAMILLONI, A. "Epistemología de la didáctica de las ciencias sociales". En Aisenberg, B. y Alderoqui S. Didáctica de las Ciencias Sociales. Aportes y Reflexiones. Buenos Aires. Paidós. 1994. Pág 27 